\title{
Matrix Differentiation of $S$-Functions
}

\author{
By H. O. Foclkes
}

(Received 26th April 1949. Read 4th June 1949.)

1. It has been shown $(1 ; 2,136)$ that if $S_{r}, a_{r}, h_{r}$ denote respectively the symmetric functions $\Sigma \lambda_{i}^{r}, \Sigma \lambda_{1} \lambda_{2} \ldots \lambda_{r}$, and the homogeneous product sum of degree $r$ of the latent roots $\lambda_{1}, \lambda_{2}, \ldots, \lambda_{n}$ of the matrix $X=\left[x_{i j}\right]$, then

$\Omega S_{r}=r X^{r-1}$,

$S 2 a_{r}=(-1)^{r-1}\left\{X^{r-1}-a_{1} X^{r-2}+a_{2} X^{r-3}-\ldots+(-1)^{r-1} a_{r-1}\right\}$,

$\Omega h_{r}=X^{r-1}+h_{1} X^{r-2}+h_{2} X^{r-3}+\ldots+h_{r-1}$,

where $\Omega=\left[\frac{\partial}{\partial x_{j i}}\right]$.

The symmetric functions known as Schur-functions, or $S$-functions, are defined $(3,82)$ in terms of group characters and are each associated with a partition of a number. In particular the $S$-functions denoted by $\left\{1^{r}\right\}$ and $\{r\}$ are respectively $a_{r}$ and $h_{r}$. Hence (2) and (3) are particular cases of a more general expression for $\Omega\{\lambda\}$ where $\{\lambda\}$ is the $S$-function corresponding to any partition $(\lambda)$. In this note an expression for $\Omega\{\lambda\}$ is obtained in the form of a polynomial in $X$ with coefficients which are linear functions of $S$-functions, and which can be found without recourse to tables of group characters.

In addition, generalisations of Turnbull's (4) theorems

and

$$
\Omega^{2} h_{r+1}=(n+r) \Omega h_{r}
$$

$$
\Omega^{2} a_{r+1}=-(n-r) \Omega a_{r},
$$

are also obtained. It is found that in the general theorems $h_{r+1}$ and $a_{r+1}$ have to be replaced by certain linear functions of $S$-functions, and the iterative property begins with a higher power of $\Omega$.

$$
\text { 2. Since } \frac{\partial S_{r}^{a}}{\partial x_{j i}}=\frac{\partial S_{r}^{a}}{\partial S_{r}} \frac{\partial S_{r}}{\partial x_{j i}}
$$

then

$$
\Omega S_{r}^{a}=\frac{\delta S_{r}^{a}}{\partial S_{r}} \Omega S_{r}
$$


Similarly $\quad \Omega\left(S_{r}^{a} S_{\varepsilon}^{b} S_{t}^{c} \cdots\right)$

$=\frac{\partial\left(S^{a}{ }_{r} S^{b}{ }_{s} S_{t}^{c} \ldots\right)}{\partial S_{r}} \Omega S_{r}+\frac{\partial\left(S_{r}^{a} S_{s}^{b} S_{t}^{c} \ldots\right)}{\partial S_{s}} \Omega S_{s}+\frac{\partial\left(S_{r}^{a} S_{s}^{b} S_{t}^{c} \ldots\right)}{\partial S_{t}^{\prime}} \Omega S_{t}+\ldots$

By definition,

$$
\{\lambda\}=\frac{1}{m !} \sum_{\rho} h_{\rho} \chi_{\rho}{ }^{(\lambda)} S_{1}^{u} S_{2}^{b} \cdots
$$

where $h_{\rho}$ is the order of the class $\rho$ determined by the partition $1^{a} 2^{b} \ldots$ of $m$, and $\chi_{\rho}{ }^{(\lambda)}$ is the characteristic of the class $\rho$ in the irreducible representation of the symmetric group associated with the partition $(\lambda)$ of $m$. Hence

$$
\begin{aligned}
\Omega\{\lambda\} & =\frac{\partial\{\lambda\}}{\partial S_{1}} \Omega S_{1}+\frac{\partial\{\lambda\}}{\partial S_{2}} \Omega S_{2}+\ldots+\frac{\partial\{\lambda\}}{\partial S_{n}} \Omega S_{n} \\
& =\frac{\partial\{\lambda\}}{\partial S_{1}}+2 \frac{\partial\{\lambda\}}{\partial S_{2}} X+3 \frac{\partial\{\lambda\}}{\partial S_{3}} X^{2}+\ldots+n{ }_{\partial S_{n}}^{\partial-\ldots} X^{n-1}
\end{aligned}
$$

The coefficients of the various powers of $X$ could be found by differentiation and expressed in terms of $S$-functions if the appropriate tables of group characters are available. They can, however, be found more easily without the use of the tables. Any operator $\partial / \partial S_{r}$ can be written as

$$
\frac{1}{j}\left[\chi_{r}{ }^{\left({ }{ }_{1}\right)} D_{\lambda_{1}}+\chi_{r}^{\left(\lambda_{2}\right)} D_{\lambda_{2}}+\ldots \chi_{r}{ }^{\left(\lambda_{j}\right)} D_{\lambda_{j}}\right]
$$

where $\chi_{r}^{(\lambda)}$ is the characteristic of the class $(r)$ of the symmetric group of order $r$ ! corresponding to the partition $\left(\lambda_{i}\right)$, and $D_{\lambda i}$ is an operator defined as (5)

$$
\sum_{\rho} \chi_{\rho}^{\left(\lambda_{i}\right)} \frac{1}{a ! b ! \ldots} \frac{\partial^{a+b+\cdots}}{\partial S_{1}^{a} \partial S_{2}^{b} \ldots} .
$$

It so happens that the characteristics of the class $(r)$ are $(-1)^{k-1}$ for every partition $\left(r-k+1,1^{k-1}\right), \quad k=1,2, \ldots, r$, and are zero for all other partitions $(6,134)$. Hence

$$
r \frac{\partial}{\partial S_{r}}=D_{r}-D_{r-1,1}+D_{r-2,1^{2}}-D_{r-3,1^{3}}+\ldots+(-1)^{r-1} D_{1^{r}} .
$$

The effect of $D_{\mu}$ on $\{\lambda\}$ is (5)

$$
D_{\mu}\{\lambda\}=\Sigma g_{\mu \nu \lambda}\{\nu\}
$$

where $g_{\mu \nu \lambda}$ is the coefficient of $\{\lambda\}$ in $\{\mu\}\{\nu\}$. Hence the coefficients in 
$\Omega\{\lambda\}$ can be found from a knowledge of products of $S$-functions, without using the tables of group characters. For example,

$\Omega\left\{2^{2} 1^{2}\right\}=D_{1}\left\{2^{2} 1^{2}\right\}+\left(D_{2}-D_{12}\right)\left\{2^{2} 1^{2}\right\} X+\left(D_{2}-D_{21}+D_{1^{3}}\right)\left\{2^{2} 1^{2}\right\} X^{2}$

$+\left(D_{4}-D_{31}+D_{21^{2}}-D_{1^{4}}\right)\left\{2^{2} 1^{2}\right\} X^{3}+\left(D_{5}-D_{41}+D_{31^{2}}-D_{21^{3}}+D_{1^{3}}\right)\left\{2^{2} 1^{2}\right\} X^{4}$ $=\left(\left\{21^{3}\right\}+\left\{2^{2} 1\right\}\right)-\left(\left\{1^{4}\right\}+\left\{2^{2}\right\}\right) X+\{2\} X^{3}-\{1\} X^{4}$.

When the number of parts in a partition exceeds $n$, the corresponding $S$-function vanishes identically $(3,91)$, and the expression for $\Omega\{\lambda\}$ then reduces to a polynomial identity in $X$, which must of course contain the characteristic function of $X$ as a factor. Thus in the above example, if $n=3$, then

$$
\{1\} X^{4}-\{2\} X^{3}+\left\{2^{2}\right\} X-\left\{2^{2} 1\right\}=0,
$$

which is equivalent to

$$
\left(X^{3}-\{1\} X^{2}+\left\{1^{2}\right\} X-\left\{1^{3}\right\}\right)\left(\{1\} X+\left\{1^{2}\right\}\right)=0 .
$$

3. To extend Turnbull's results

$$
\begin{aligned}
& \Omega^{2}\{p\}=(n+p-1) \Omega\{p-1\}, \\
& \Omega^{2}\left\{1^{q}\right\}=-(n-q+1) \Omega\left\{1^{q-1}\right\}
\end{aligned}
$$

a stage further, operate twice with $\Omega$ on the relation

$$
\left\{p 1^{q}\right\}+\left\{p+1,1^{q-1}\right\}=\{p\}\left\{1^{q}\right\}
$$

obtaining

$$
\Omega\left\{p 1^{q}\right\}+\Omega\left\{p+1,1^{q-1}\right\}=\{p\} \Omega\left\{1^{q}\right\}+\left\{1^{q}\right\} \Omega\{p\}
$$

and

$$
\begin{aligned}
& \Omega^{2}\left\{p 1^{q}\right\}+\Omega^{2}\left\{p+1,1^{q-1}\right\}=2 \Omega\{p\} \Omega\left\{1^{q}\right\}+\{p\} \Omega^{2}\left\{1^{q}\right\}+\left\{1^{q}\right\} \Omega^{2}\{p\} \\
= & 2 \Omega\{p\} \Omega\left\{1^{q}\right\}-(n-q+1)\{p\} \Omega\left\{1^{q-1}\right\}+(n+p-1)\left\{1^{q}\right\} \Omega\{p-1\} . \quad(6)
\end{aligned}
$$

When $q=1$, this becomes

$$
\begin{aligned}
\Omega^{2}\{p 1\} & =2 \Omega\{p\}+(n+p-1)\{1\} \Omega\{p-1\}-(n+p) \Omega\{p\} \\
& =(n+p-1)\{1\} \Omega\{p-1\}-(n+p-2) \Omega\{p\} .
\end{aligned}
$$

Again applying $\Omega$, we have

$$
\begin{aligned}
\Omega^{3}\{p 1\}= & (n+p-1)[\Omega\{p-1\}+(n+p-2)\{1\} \Omega\{p-2\}] \\
& -(n+p-2)(n+p-1) \Omega\{p-1\} \\
= & (n+p-1)[(n+1 p-2)\{1\} \Omega\{p-2\}-(n+p-3) \Omega\{p-1\}] \\
= & (n+p-1) \Omega^{2}\{p-1,1\} .
\end{aligned}
$$


Hence (4), which may be written as

$$
\Omega^{r}\{p\}=(n+p-1) \Omega^{r-1}\{p-1\}, \quad p>0, r \geqq 2,
$$

has as an extension the theorem that

$$
\Omega^{r}\{p l\}=(n+p-1) \Omega^{r-1}\{p-1,1\}, \quad p=1, r \geqq 3 .
$$

Similarly, by taking $p=1$ in (6), we have

$$
\Omega^{2}\left\{2,1^{q-1}\right\}=(n-q+2) \Omega\left\{1^{q}\right\}-(n-q+1)\{1\} \Omega\left\{1^{q-1}\right\}
$$

and

$$
\begin{aligned}
& \Omega^{3}\left\{2,1^{q-1}\right\}=-(n-q+2)(n-q+1) \Omega\left\{1^{q-1}\right\} \\
& -(n-q+1)\left[\Omega\left\{1^{q-1}\right\}-(n-q+2)\{1\} \Omega\left\{1^{q-2}\right\}\right] \\
& =-(n-q+1)\left[(n-q+3) \Omega\left\{1^{q-1}\right\}-(n-q+2)\{1\} \Omega\left\{1^{q-2}\right\}\right] \\
& =-(n-q+1) \Omega^{2}\left\{2,1^{q-2}\right\} \text {. }
\end{aligned}
$$

If $\left\{2,1^{q-1}\right\}$ is written as $\left\{1^{q} 1\right\}$ in order to bring the form of the result into correspondence with (7), then (5), written as

$\Omega^{r}\left\{1^{q}\right\}=-(n-q+1) \Omega^{r-1}\left\{1^{q-1}\right\}, \quad q>0, r \geqq 2$,

has as an extension the theorem that

$$
\Omega^{r}\left\{1^{q} 1\right\}=-(n-q+1) \Omega^{r-1}\left\{1^{q-1} 1\right\}, \quad q>1, r \geqq 3 .
$$

Taking $q=2$ and $p=2$ in (6) does not lead to corresponding expressions for $\Omega^{r}\left\{p 1^{2}\right\}$ and $\Omega^{r}\left\{1^{q} 1^{2}\right\}$, but the simple recursive property shown in (4), (5), (7), (8) appears again in the results of the next section, in which a new type of operand is considered.

4. Consider the expressions defined as

$$
\begin{aligned}
& (p, 0)=\{p\}, \quad p>0, \\
& (p, 1)=\{p 1\}, \quad p>1, \\
& (p, 2)=\{p 2\}+\left\{p 1^{2}\right\}, \quad p>2, \\
& (p, 3)=\{p 3\}+2\{p 21\}+\left\{p 1^{3}\right\}, \quad p>3, \\
& (p, 4)=\{p 4\}+3\{p 31\}+2\left\{p 2^{2}\right\}+3\left\{p 21^{2}\right\}+\left\{p 1^{4}\right\}, \quad p>4,
\end{aligned}
$$

in which the coefficient of $\left\{p \lambda_{1} \lambda_{2} \lambda_{3} \ldots\right\}$ in $(p, m)$ is the characteristic of the class $\left(1^{m}\right)$ in the irreducible representation of the symmetric group of order $m$ ! corresponding to the partition $\left(\lambda_{1} \lambda_{2} \lambda_{3} \ldots\right)$ of $m$. The set of coefficients can be written down at once from the character table, as they constitute the first column of the table as usually presented (3). 
These expressions are such that

$\{1\}(p, m)=(p, m+1)+(p+1, m)$.

\section{Hence}

$\Omega(p, m+1)=(p, m)+\{1\} \Omega(p, m)-\Omega(p+1, m)$,

$\Omega^{2}(p, m+1)=2 \Omega(p, m)+\{1\} \Omega^{2}(p, m)-\Omega^{2}(p+1, m)$,

$\Omega^{3}(p, m+1)=3 \Omega^{2}(p, m)+\{1\} \Omega^{3}(p, m)-\Omega^{3}(p+1, m)$,

$\Omega^{r}(p, m+1)=r \Omega^{r-1}(p, m)+\{1\} \Omega^{r}(p, m)-\Omega^{r}(p+1, m)$.

From (4), $\Omega^{2}(p, 0)=(n+p-1) \Omega(p-1,0)$, and so for $m=0$ $\Omega^{2}(p, 1)=(n+p-1)\{1\} \Omega(p-1,0)-(n+p-2) \Omega(p, 0)$, $\Omega^{3}(p, 1)=(n+p-1)[(n+p-2)\{1\} \Omega(p-2,0)-(n+p-3) \Omega(p-1,0)]$ $=(n+p-1) \Omega^{2}(p-1,1)$,

which is (7). For $m=1$,

$$
\begin{aligned}
& \Omega^{4}(p, 2)=4 \Omega^{3}(p, 1)+\{1\} \Omega^{4}(p, 1)-\Omega^{4}(p+1,1) \\
& =3 \Omega^{3}(p, 1)+(n+p-1)\{1\} \Omega^{3}(p-1,1)-(n+p) \Omega^{3}(p, 1)+\Omega^{3}(p, 1) \\
& =(n+p-1)\left[3 \Omega^{2}(p-1,1)+\{1\} \Omega^{3}(p-1,1)-\Omega^{3}(p, 1)\right] \\
& =(n+p-1) \Omega^{3}(p-1,2) .
\end{aligned}
$$

The generalisation is now evident and the proof follows by induction. If $\Omega^{r}(p, m)=(n+p-1) \Omega^{r-1}(p-1, m)$ for $r \geqq m+2$, then $\Omega^{r}(p, m+1)=(r-1) \Omega^{r-1}(p, m)+\{1\} \Omega^{r}(p, m)-\Omega^{r}(p+1, m)$

$=(n+p-1)\left[(r-1) \Omega^{r-2}(p-1, m)+\{1\} \Omega^{r-1}(p-1, m)-\Omega^{r-1}(p, m)\right]$ for $r-1 \geqq m+2$, $=(n+p-1) \Omega^{r-1}(p-1, m+1), \quad$ for $r \geqq m+3$.

5. A corresponding result can be obtained for the conjugate partitions. For convenience in notation write the conjugate of $\left\{q \lambda_{1} \lambda_{2} \ldots\right\}$ as $\left\{1^{q} \mu_{1} \mu_{2} \ldots\right\}$, where $\left\{\mu_{1} \mu_{2} \ldots\right\}$ and $\left\{\lambda_{1} \lambda_{2} \ldots\right\}$ are conjugates. Then the expressions

$$
\begin{aligned}
& \left(1^{q}, 0\right)=\left\{1^{q}\right\}, \quad q>0, \\
& \left(1^{q}, 1\right)=\left\{1^{q} 1\right\}, \quad q>1, \\
& \left(1^{q}, 2\right)=\left\{1^{q} 2\right\}+\left\{1^{q} 1^{2}\right\}, \quad q>2, \\
& \left(1^{q}, 3\right)=\left\{1^{q} 3\right\}+2\left\{1^{q} 21\right\},+\left\{1^{q} 1^{3}\right\}, \quad q>3, \\
& \left(1^{q}, 4\right)=\left\{1^{q} 4\right\}+3\left\{1^{q} 31\right\}+2\left\{1^{q} 2^{2}\right\}+3\left\{1^{q} 21^{2}\right\}+\left\{1^{q} 1^{q}\right\}, \quad q>4
\end{aligned}
$$


are such that

$\{1\}\left(1^{q}, m\right)=\left(1^{q}, m+1\right)+\left(1^{q+1}, m\right)$.

\section{Hence}

$\Omega^{r}\left(1^{q}, m+1\right)=r \Omega^{r-1}\left(1^{q}, m\right)+\{1\} \Omega^{r}\left(1^{q}, m\right)-\Omega^{r}\left(1^{q+1}, m\right)$.

Assume

$\Omega^{r}\left(1^{q}, m\right)=-\left(n-q^{*}+1\right) \Omega^{r-1}\left(1^{q-1}, m\right) \quad$ for $r \geqq m+2$.

Then

$\Omega^{r}\left(1^{q}, m+1\right)=(r-1) \Omega^{r-1}\left(1^{q}, m\right)+\{1\} \Omega^{r}\left(1^{q}, m\right)$

$-\Omega^{r}\left(1^{q+1}, m\right)+\Omega^{r-1}\left(1^{q}, m\right)$

$=-(n-q+1)\left[(r-1) \Omega^{r-2}\left(1^{q-1}, m\right)+\{1\} \Omega^{r-1}\left(1^{q-1}, m\right)\right.$

$$
\left.-\Omega^{r-1}\left(1^{g}, m\right)\right] \quad \text { for } r-1 \geqq m+2
$$

$=-(n-q+1) \Omega^{r-1}\left(1^{q-1}, m+1\right), \quad$ for $r \geqq m+3$.

Since $m=0$ gives

$\Omega^{r}\left(1^{q}, 0\right)=-(n-q+1) \Omega^{r-1}\left(1^{q-1}, 0\right) \quad$ for $r \geqq 2$,

which is known to be true by (5), then the result is proved by induction.

\section{REFERENCES.}

(1) H. W. Turnbull, "On differentiating a matrix," Proc. Edinburgh Maih. Soc. (2), 1 (1927), 111-128.

(2) J. H. M. Wedderburn, Lectures on matrices (New York, 1934).

(3) D. E. Littlewood, Group characters and matric representations of groups (Oxford, 1940).

(4) H. W. Turnbull, "Matrix differentiation of the characteristic function," Proc. Edinburgh Math. Soc. (2), 2 (1931), 256-264.

(5) H. O. Foulkes, "Differential operators associated with S-functions," Journal London Muth. Soc., 24 (1949), 136-143.

(6) F. D. Murnaghan, Theory of group representations (Baltimore, 1938).

\section{UNIVERSITY COLXEGE,}

Swansea. 